\title{
Case report: Desmopressin and somnambulism
}

\author{
Charlotte Van Herzeele*, Karlien Dhondt, Johan Vande Walle \\ University Hospital Ghent, Ghent, Belgium \\ Email: ${ }^{*}$ charlotte.vanherzeele@ugent.be
}

Received 11 June 2013; revised 13 July 2013; accepted 21 July 2013

Copyright (C) 2013 Charlotte Van Herzeele et al. This is an open access article distributed under the Creative Commons Attribution License, which permits unrestricted use, distribution, and reproduction in any medium, provided the original work is properly cited.

\begin{abstract}
Background: Nocturnal enuresis is caused by a mismatch between nocturnal urine production and bladder capacity. Together with a presumed decreased arousability, this results in an inability to awaken in response to a full bladder. According to recent findings, a disrupted sleep might play a role in the pathophysiology of enuresis. Case: A 7-year-old boy was diagnosed with primary nocturnal enuresis caused by nocturnal polyuria and a concomitant parasomnia, somnambulism. A polysomnographic study was performed before treating the nocturnal enuresis with the oral lyophylisate formulation of desmopressin (melt). After 1 day of treatment, both nocturnal enuresis and somnambulism disappeared. Treatment was ceased after six months. One week later, the child started to produce more urine and redeveloped nocturnal polyuria. Somnambulism reappeared followed by nocturnal enuresis two weeks later. The same treatment protocol was started up, resulting in disappearance of both nocturnal enuresis and somnambulism. Conclusion: This case report documents the beneficial effect of desmopressin melt on both nocturnal enuresis, caused by nocturnal polyuria, and a concomitant parasomnia in particularly somnambulism.
\end{abstract}

Keywords: Children; Enuresis; Bedwetting; Somnambulism; Sleep; Sleepwalking; Desmopressin; Nocturnal Polyuria; Parasomnia

\section{INTRODUCTION}

Monosymptomatic nocturnal enuresis (MNE) is caused by a mismatch between the nocturnal diuresis volume and the bladder capacity. It was widely accepted that a decreased arousability, provokes an inability to awaken in response to a full bladder [1]. According to recent

*Corresponding author. findings, disrupted sleep rather than decreased arousability might play an important role in the pathophysiology of nocturnal enuresis (NE) [2]. Sleep of children with NE is characterized by sleep fragmentation. Even more, children with NE clearly have more daytime sleepiness and suffer from sleep deprivation [3]. Mahler et al. demonstrated that sleep deprivation might result in increased nocturnal diuresis volume [4]. During polysomnography, our study group observed increased periodic limb movements (PLMS) and cortical arousals in children with refractory NE [5]. The association among PLMS, arousals and NE still remains unclear.

Nocturnal polyuria (NP) is related to a deficient vasopressin (AVP) secretion overnight. Therefore, the synthetic AVP analogue desmopressin is widely used to treat children with MNE [6]. Its antidiuretic action results from an increased renal reabsorption of water, leading to a reduced volume and more concentrated urine. Desmopressin is recommended by the International Consultation on Incontinence (Grade A, Level 1) for the treatment of MNE.

Somnambulism (sleepwalking) is a well-known sleep disorder, classified as a parasomnia. Electro-encephalographic recording during this event is characterized by a cortical arousal that emerges during the first third of the night when slow wave sleep is present $[7,8]$. This sudden change in EEG frequency can be caused by PLMS [8] or obstructive sleep apnea [9] or can occur due to brain maturation [10]. Hublin et al. demonstrated retrospectively by questionnaires that $7.7 \%$ men and $9.7 \%$ women reported sleepwalking "often" or "sometimes". $17.9 \%$ of the men and $17.0 \%$ of the women reported "a few times" sleepwalking during childhood (4 - 15 years) [11]. According to Esposito et al. somnambulism is far more presented in children with NE (22.1\%) compared to healthy controls (6.7\%) [12].

In the following case report we demonstrate a comorbidity of NE and the parasomnia sleepwalking, both resolved by an effective treatment of enuresis. 


\section{CASE REPORT}

The boy was six years old when his parents consulted a child nephrologist. He experienced diurnal incontinence once a month, daily wet urine spots in underpants, urge incontinence with squatting and giggling incontinence. Moreover, NE was present almost every night. Home recordings revealed that the child had small voided volumes, although drinking schedule was appropriate. NE usually took place during the first hours of the night with documented NP.

After successful treatment of the daytime symptoms (urotherapy, oxybutinin), the picture of NE changed. The boy started "bed-wetting" during sleepwalking episodes. His mother described it as follows: "it looks like he was searching for a toilet and when he thought he found one, he started to pee". The combination of sleepwalking and "wetting" persisted for a year when the boy and his mother consulted again. Screening showed no longer daytime symptoms. There was a normal bladder volume, but NP still persisted.

The boy got subsequent a video-polysomnographic study. Sleepwalking was not present the night of the polysomnography, but PLMS were clearly observed. After the polysomnography $120 \mu \mathrm{g}$ of the oral lyophylisate formulation of desmopressin (melt) was given. Not only NP disappeared but both sleepwalking and NE ceased. One night he forgot to take his medication and that night he experienced again NE.

After six months of successful treatment, desmopressin melt was stopped. One week later, the child started to produce more urine and developed NP. His mother reported a more disrupted sleep. Two weeks later the boy started sleepwalking again and after three weeks NE during sleepwalking recurred. He was treated again with desmopressin melt and once more both sleepwalking and NE stopped.

\section{DISCUSSION}

The recurrence of both enuresis and somnambulism due to the discontinuation of desmopressin melts and the resumption of benefits due to the restart of the drug suggests that the desmopressin therapy not only has an antienuretic effect [13], but is also responsible for the disappearance of the somnambulism. The positive effect on the somnambulism is likely to be related to the antidiuretic effect. We speculate that the boy suffering from NP without desmopressin, experienced a sense of a full bladder provoking cortical arousals in his sleep. These arousals result in a parasomnia, in this case: somnambulism. This incomplete awakening while searching for a toilet, was previously suggested by Yeung et al. [2]. By using desmopressin, the child no longer had NP and therefore did not experience arousal stimuli from the bladder while sleeping. Probably, as a result, no interrupting arousals caused the parasomnia at that time.

However, a direct effect on the central nervous system (CNS) by desmopressin can not entirely be excluded [14, 15]. The major mechanism of desmopressin in NE results in an increased urinary concentration and subsequent decreased diuresis-rate. Even though, the exact mechanism of our observation is not yet clear.

This case study emphasizes again the important comorbidity of a disrupted sleep (somnambulism, PLMS, cortical arousals) and NE together with NP. Yet, a prospective study is needed to further elucidate the pathophysiologic mechanisms involved.

\section{REFERENCES}

[1] Hjalmas, K., Arnold, T., Bower, W., et al. (2004) Nocturnal enuresis: An international evidence based management strategy. Journal of Urology, 171, 2545-2561. doi:10.1097/01.ju.0000111504.85822.b2

[2] Yeung, C.K., Diao, M. and Sreedhar, B. (2008) Cortical arousal in children with severe enuresis. The New England Journal of Medicine, 358, 2414-2415. doi:10.1056/NEJMc0706528

[3] Cohen-Zrubavel, V., Kushnir, B., Kushnir, J. and Sadeh, A. (2011) Sleep and sleepiness in children with nocturnal enuresis. Sleep, 34, 191-194.

[4] Mahler, B., Kamperis, K., Schroeder, M., et al. (2012) Sleep deprivation induces excess diuresis and natriuresis in healthy children. American Journal of PhysiologyRenal Physiology, 302, F236-F243. doi:10.1152/ajprenal.00283.2011

[5] Dhondt, K., Raes, A., Hoebeke, P., et al. (2009) Abnormal sleep architecture and refractory nocturnal enuresis. Journal of Urology, 182, 1961-1965. doi:10.1016/j.juro.2009.05.103

[6] Neveus, T. (2006) The evaluation and treatment of therapy-resistant enuresis: A review. Upsala Journal of Medical Sciences, 111, 61-71. doi:10.3109/2000-1967-026

[7] Guilleminault, C., Lee, J.H., Chan, A., et al. (2005) NonREM-sleep instability in recurrent sleepwalking in prepubertal children. Sleep Medicine, 6, 515-521. doi:10.1016/j.sleep.2005.03.003

[8] Guilleminault, C., Palombini, L., Pelayo, R. and Chervin, R.D. (2003) Sleepwalking and sleep terrors in prepubertal children: What triggers them? Pediatrics, 111, e17-e25. doi:10.1542/peds.111.1.e17

[9] Guilleminault, C., Poyares, D., Rosa, A. and Huang, Y.S. (2005) Heart rate variability, sympathetic and vagal balance and EEG arousals in upper airway resistance and mild obstructive sleep apnea syndromes. Sleep Medicine, 6, 451-457. doi:10.1016/j.sleep.2005.03.014

[10] Campbell, I.G., Grimm, K.J., de Bie, E. and Feinberg, I. (2012) Sex, puberty, and the timing of sleep EEG measured adolescent brain maturation. Proceedings of the $\mathrm{Na}$ tional Academy of Sciences of the United States of America, 109, 5740-5743. doi:10.1073/pnas.1120860109 
[11] Hublin, C., Kaprio, J., Partinen, M., Heikkila, K. and Koskenvuo, M. (1997) Prevalence and genetics of sleepwalking: A population-based twin study. Neurology, 48, 177-181. doi:10.1212/WNL.48.1.177

[12] Esposito, M., Gallai, B., Parisi, L., et al. (2013) Primary nocturnal enuresis as a risk factor for sleep disorders: An observational questionnaire-based multicenter study. Journal of Neuropsychiatric Disease and Treatment, 9, 437-443.

[13] Glazener, C.M.A. and Evans, J.H.C. (2002) Desmopressin for nocturnal enuresis in children. Cochrane Database of Systematic Reviews, Article ID: CD002112.
[14] Schulz-Juergensen, S., Rieger, M., Schaefer, J., Neusuess, A. and Eggert, P. (2007) Effect of 1-desamino-8-D-arginine vasopressin on prepulse inhibition of startle supports a central etiology of primary monosymptomatic enuresis. Journal of Pediatrics, 151, 571-574. doi:10.1016/j.jpeds.2007.05.024

[15] Rahm, C., Schulz-Juergensen, S. and Eggert, P. (2010) Effects of desmopressin on the sleep of children suffering from enuresis. Acta Paediatr, 99, 1037-1041. doi:10.1111/j.1651-2227.2010.01724.x

\section{ABBREVIATIONS}

MNE: monosymptomatic nocturnal enuresis;

NE: nocturnal enuresis;

PLMS: periodic limb movements of sleep;

NP: nocturnal polyuria;

AVP: arginine vasopressin;

EEG: electroencephalography;

CNS: central nervous system. 